\title{
Guinea Pigs Naturally Infected by Leishmania enriettii: Clinical Analyses, Parasite Isolation and Identification
}

\author{
Vanete Thomaz Soccol ${ }^{1 *}$ \\ https://orcid.org/0000-0001-9301-541X
}

Edilene Alcântara de Castro²

https://orcid.org/0000-0002-1678-5530

Larissa Reifur ${ }^{2}$

https://orcid.org/0000-0003-0536-8915
Valéria Natascha Teixeira ${ }^{3}$
https://orcid.org/0000-0003-4831-3463

Rogério Ribas Lange 4

https://orcid.org/0000-0003-2262-4474

${ }^{1}$ Federal University of Paraná, Graduate Program in Bioprocess Engineering and Biotechnology, Curitiba, Paraná, Brazil; ${ }^{2}$ Federal University of Paraná, Basic Pathology Department, Curitiba, Paraná, Brazil; ${ }^{3}$ Catholic University of Paraná, Curitiba, Paraná, Brazil; ${ }^{4}$ Federal University of Paraná, Veterinary Medicine Department, Curitiba, Paraná, Brazil.

Editor-in-Chief: Alexandre Rasi Aoki

Associate Editor: Alexandre Rasi Aoki

Received: 2021.02.19; Accepted: 2021.08.25.

*Correspondence: vanetesoccol@gmail.com; Tel.: +55-41-999565001(V.T.S.).

\section{HIGHLIGHTS}

- Leishmania enriettii is revisited.

- Natural infection of Leishmania enriettii.

- Dispersion of this enigmatic Leishmania species.

- Leishmania enriettii cause diffuse leishmaniosis.

Abstract: Leishmania enriettii has only been found in Curitiba metropolitan region, southern Brazil were it was first observed in Cavia porcellus from the vivarium of Instituto de Biologia e Pesquisas Tecnológicas (IBPT - today named TECPAR) by Medina, 1944. Despite more than a half century from its discovery and several research articles on this species, the natural clinical signs in guinea pigs and the parasite genetic variability is still unclear. The aims of this study were to describe the clinical features, investigate the potential wild reservoirs and, in addition, we intended to understand the polymorphism trait of the species. We analyzed 26 naturally infected guinea pigs from eight Paraná state cities. All animals showed lesions compatible with leishmaniosis, such as skin nodules or ulcers on body extremities. Direct examination of the lesion samples obtained by fine-needle aspiration or punch biopsy was conducted followed by isolation and identification of parasite DNA by random amplification of polymorphic DNA (RAPD)-PCR. Through the direct exam, a large number of intracellular amastigote forms were observed in the lesions. Different strains of the parasite, isolated from the 26 animals, were grouped in 5 clusters of approximately $65 \%$ similarity. We looked for $L$. enriettii in other potential reservoir hosts but the parasite was not observed. These results confirm that distinct strains of $L$. enriettii circulate in guinea pigs from Paraná state, more specifically in the Atlantic forest region, where we believe it serves as the center for dispersion of the species.

Keywords: Leishmania enriettii; diffuse cutaneous leishmaniosis; phenetic analysis; Cavia porcellus. 


\section{GRAPHICAL ABSTRACT}
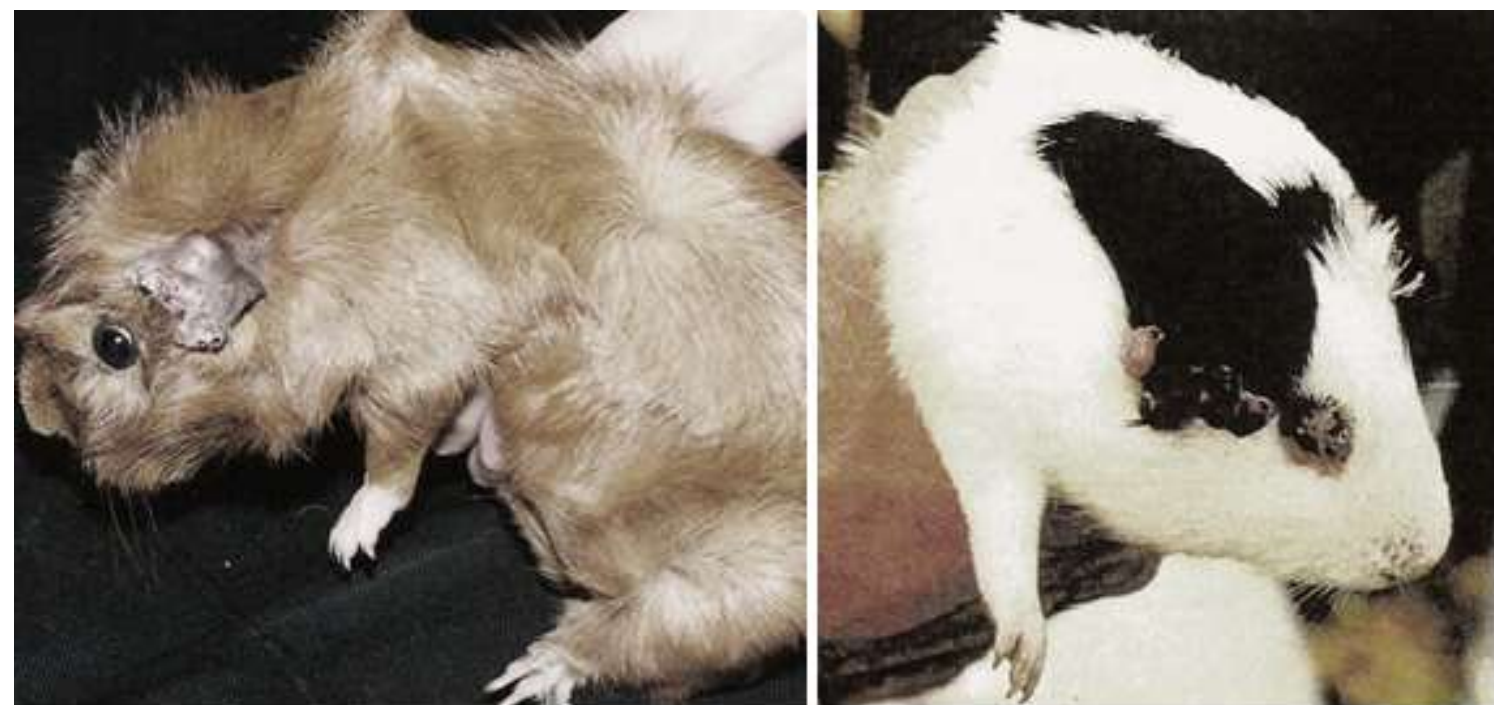

\section{INTRODUCTION}

Medina detected Leishmania (Leishmania) enriettii infecting guinea pigs from Paraná state in 1944 [1] and together with Muniz, they described and nominated the parasite four years later [2]. This first isolated strain of the parasite was distributed to laboratories worldwide and today sold by ATCC ${ }^{\circledR}$ Number: $\underline{50120^{\mathrm{TM}}}$. Leishmania enriettii has been used as a model organism to several studies such as experimental infections, cell mediated immune studies, intracellular Leishmania survival studies, as a model to study the dissemination of cutaneous leishmaniosis, disease treatment and more recently to genome of the parasite ([3-14] to cite a few).

Despite all the experimental work using $L$. enriettii as a model organism, very few are the studies showing the natural infection since the description of the species. Only after 19 years since $L$. enriettii description, Luz and coauthors, 1967 reported the reappearance of spontaneous leishmaniosis in guinea pigs, caused by $L$. enriettii, in Curitiba outskirts, Paraná State-Brazil [15]. The authors also searched for the potential vectors and found Lutzomyia monticola (Costa Lima, 1932) and L. correalimai (Martins, Coutinho \& Luz, 1970) in the same area [15]. In 1994, the species was found in naturally infected guinea pigs, but now in São Paulo State, in a rural area from Capão Bonito district, near the Atlantic forest [16]. Two years later, three cases of cutaneous leishmanosis in guinea pigs from a rural area near Curitiba (Paraná state) were reported by Thomaz Soccol and coauthors, 1996 [17]. These authors isolated the parasite and identified L. enriettii through isoenzyme analyses. Two of the three cases were $100 \%$ similar to the initially described parasite and the third isolate was a new strain [17].

Currently, the output from searching L. enriettii using open databases is of approximately 2,940 hits by Google scholar, 162 on Pubmed, and 521 registered patents on google patents. Regardless of all the work, there is a lack of information on the epidemiology of this species and on the authenticity of the first strain stored in different cryobanks.

It is accepted that $L$. enriettii exists and survives in guinea pigs from Brazil. However, its geographical distribution; the clinical signs occurring from a natural infection and its genetic variability have not been explored. In this study, we used pet owned guinea pigs and captured wild animals from distinct areas within the Paraná state in search for different strains of $L$. enriettii. The purpose was to contribute to the better knowledge of the taxon's polymorphism, geographic distribution, and pathogenesis caused by the natural infection. 


\section{MATERIAL AND METHODS}

\section{Study area}

The guinea pigs presenting skin lesions and the other wild animals were from eight Paraná cities, including Laranjeiras do Sul, Castro, Curitiba, Campina Grande do Sul, Pinhais, Quatro Barras, Almirante Tamandaré, and Mandirituba (Figure 1).

\section{Guinea pigs}

Twenty six guinea pigs (Cavia porcellus) obtained from different pet owners or from breeders showing skin lesions, nodules or ulcers resembling those of cutaneous leishmaniosis were enrolled in this study. From each animal, samples from a typical lesion were obtained by punch biopsy $(4 \mathrm{~mm})$ or fine-needle aspiration or lesion scraping.

All animals were clinically examined and the progression of the clinical signs was followed up for several days or months until the animal's death. All animals eventually died and a necropsy was performed and samples from liver, lymph nodes, and spleen were taken.

\section{Reservoir research}

To determine possible wild reservoirs, 77 wild animals captured from areas listed in Fig. 1, including opossums (Didelphis albiventris), porcupines (Sphiggurus villosus), agoutis (Dasyprocta azarae), capybaras (Hydrochoerus hydrochaeris), nutrias (Myocastor coypus), and the Brazilian guinea pig (Cavia aperea) were included in the study even knowing that none of them were found with skin lesions. From these animals a blood sample, a skin biopsy fragment or fragments from other internal tissues were taken (authorization 108/03 - IBAMA, the Brazilian Institute for the Environment and Renewable Natural Resources). The aim was to isolate the parasite and contribute to the search for the potential reservoir.

\section{Direct exam}

The material obtained from guinea pigs aspirates was spread out on slides and the material from the biopsies or the internal tissue samples were transferred to a slide by imprint. The slides were stained with May-Grünwald Giemsa, and observed under the optic microscope at $1000 \mathrm{x}$.

\section{Leishmania isolation and growth}

The material (from aspirates, punch biopsies, sample from internal tissues) obtained from the 26 guinea pigs and 77 wild animals were used to isolate the parasite. The samples were macerated, if needed, and homogenized with $0.9 \%$ saline and antibiotic (penicillin $20.000 \mathrm{UI}$ and streptomycin $100 \mu \mathrm{g} / \mathrm{mL}$ ). Then, 0.5 $\mathrm{mL}$ was inoculated in the McNeal, Novy, and Nicolle (NNN) biphasic media and maintained at $24{ }^{\circ} \mathrm{C}$ for several days, as previously described [19]. At the 5th and 7th days, a small sample was observed under the microscope and if parasites were detected the culture was diluted in sterile saline, at a 1:1 (volume to volume) ratio. A volume of $0.5 \mathrm{~mL}$ was transferred to a new tube containing NNN media. At this stage, the parasites were dividing as promastigotes. If no promastigotes were seen at the 7th day, the sample was still kept and split four more times with a seven day interval. After this period and if no parasites were found, the sample was considered negative and discarded. For parasite identification the positive samples were transferred to tissue culture flasks containing RPMI 1640 media and 10\% fetal calf serum as previously described [20]. The promastigotes were then harvested during the exponential growth phase for DNA extraction.

\section{Reference strains}

The reference strains (previously identified by isoenzyme analyses) were all New World Leishmania species obtained from the Montpellier (France) cryobank: L. braziliensis (MHOM/BR/75/M2903); $L$. amazonensis (IFLA/BR/67/PH-8); L. infantum, MHOM/BR/74/PP75; L. enriettii, MCAV/BR/45/LEM99. The DNA from all these strains were extracted and used as controls and for comparisons in the RAPD technique. 


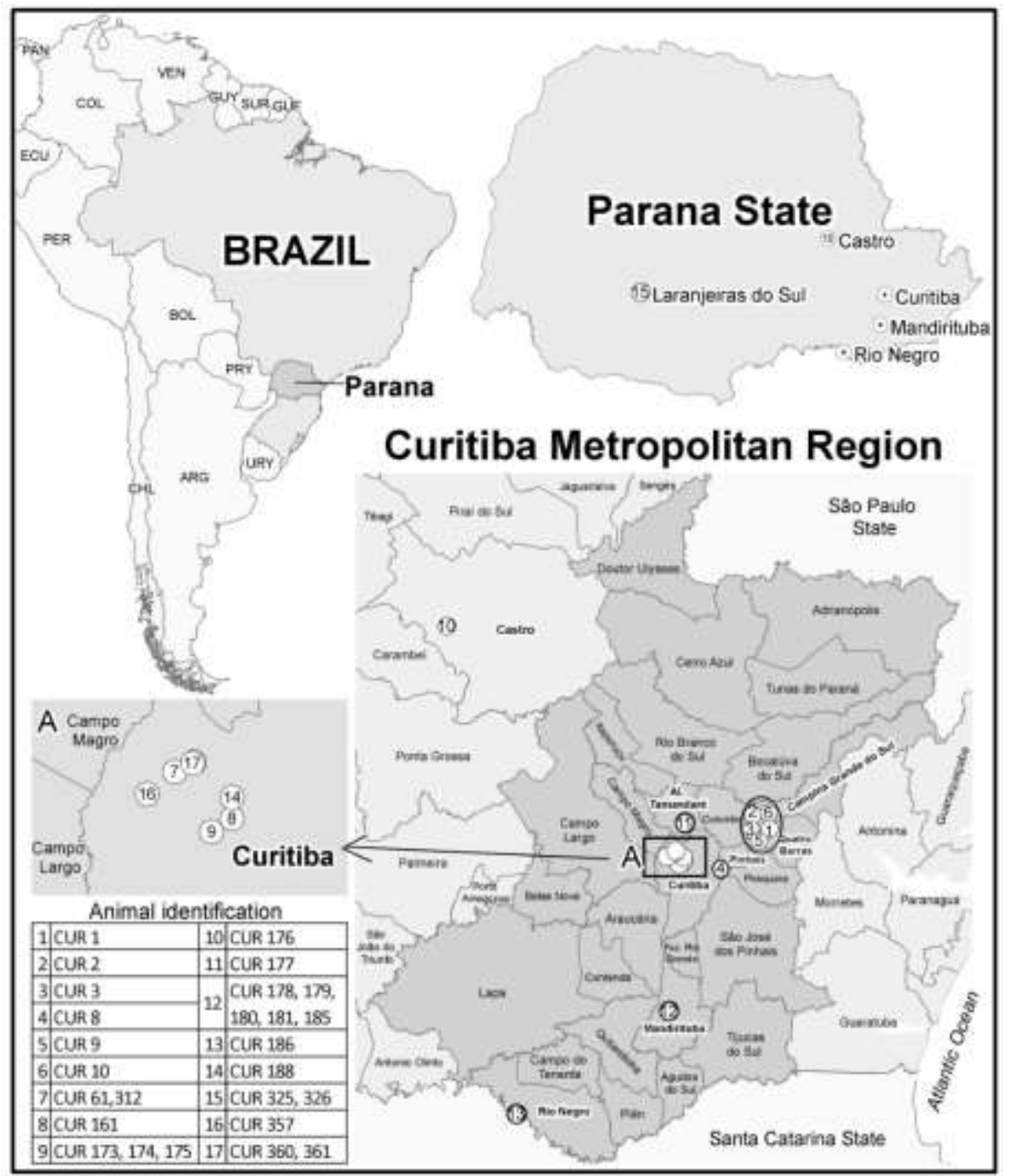

Figure 1. Distribution of guinea pigs (Cavia porcellus) with Leishmania enriettii lesions. The map on the top right corner shows Paraná state location within Brazil. In the Paraná State map, the towns where the infected guinea pigs were found at are depicted, such as Laranjeiras do Sul, Castro, and Curitiba. Within Curitiba metropolitan region (dark gray) infected animals were found in Campina Grande do Sul, Pinhais, Quatro Barras, Almirante Tamandaré, and Mandirituba. Each circled number refers to an animal where $L$. enriettii was isolated from.

\section{DNA extraction}

The promastigotes from all samples, including from the reference strains, were harvested from a $100 \mathrm{~mL}$ RPMI axenic culture through centrifugations at $4{ }^{\circ} \mathrm{C}$ in sterile $15 \mathrm{~mL}$ Falcon tubes for $20 \mathrm{~min}$ at 10,000 $\mathrm{g}$. The pellet was washed twice in $0.3 \% \mathrm{NaCl}_{2}$, suspended in a minimum volume of the same solution, and transferred to a pre-weighted $1.5 \mathrm{~mL}$ eppendorf tube. The suspension was centrifuged at $9000 \times \mathrm{g}$, for $5 \mathrm{~min}$ at $4{ }^{\circ} \mathrm{C}$, the supernatant was removed and the pellet was weighed and immediately stored at the $-20{ }^{\circ} \mathrm{C}$ freezer. The DNA extraction was conducted according to Sambrook and Russel [21] with minor modifications. The extracted DNA was suspended in $100 \mu \mathrm{L}$ of ultrapure sterile water, left at $4{ }^{\circ} \mathrm{C}$ overnight, and stored at $80{ }^{\circ} \mathrm{C}$. The DNA quantification was conducted after measuring the absorbance at $260 \mathrm{~nm}$ using a spectrophotometer (Gene Quant@).

\section{Random Amplification of Polymorphic DNA (RAPD)-PCR}

The genomic DNA extracted as described above was amplified through the RAPD technique as follows. The amplification reaction was conducted in $25 \mu \mathrm{L}$ containing $10 \mathrm{mM}$ Tris- $\mathrm{HCl}, \mathrm{pH} 8.3,50 \mathrm{mM} \mathrm{KCl}, 1.5 \mathrm{mM}$ $\mathrm{MgCl}_{2}, 0.2 \mathrm{mM}$ of each dNTP, 10 pmol of the specific primer (A2, A3, A9, or A10), $10 \mathrm{ng}$ of the genomic DNA and $2.5 \mathrm{U}$ of Taq DNA polymerase (Invitrogen). The PCR steps consisted of initial denaturation at $94^{\circ} \mathrm{C}$ for $5 \mathrm{~min}$, followed by 45 cycles of denaturation at $94^{\circ} \mathrm{C}$ for $1 \mathrm{~min}$, annealing at $36^{\circ} \mathrm{C}$ for $1 \mathrm{~min}$, and extension at $72{ }^{\circ} \mathrm{C}$ for $2 \mathrm{~min}$. The final step included an extension at $72^{\circ} \mathrm{C}$, allowed for $7 \mathrm{~min}$. The amplified products 
were separated by electrophoresis in a $1.6 \%$ agarose gel, visualized and photographed under an ultraviolet light transilluminator after ethidium bromide staining. The RAPD reaction was conducted in separate for the 4 different primers (A2- 5'TGCCGAGCTG3'; A3 5'AGTCAGCCAC3'; A9 5' GGGTAACGCC3'; and A10 5' GTGATCGCAG3'). These primers were used previously for Leishmania study [22]. The isolated strains were named with a code that followed the World Health Organization recommendations (CUR, followed by the number of the animal) and cryopreserved in liquid nitrogen, at $-196^{\circ} \mathrm{C}$.

\section{Phenetic analysis}

RAPD fingerprints obtained with each primer from all samples were captured and analyzed with the GelPro Analyzer program (Media Cybernetics) suited for fingerprint comparison and management. Only clear and reproducible bands were included in the analyses. Each band was coded to a binary matrix by denoting " 0 " or " 1 " intended for absence and presence of the band, respectively. After all bands were transferred to the matrix, the data were used as input for calculations of the genetic distances between the strains. The estimates for the genetic distances based Nei similarity coefficients were calculated using the FAMD program $[23,24]$. All the other analyses were conducted using the PAUP*4b10 program [25].

\section{RESULTS}

\section{Guinea pigs}

Among the 26 guinea pigs selected with lesions, $70 \%$ were female, all of them presented at least one skin lesion, and some had up to nine lesions in distinct sites (Table 1).

Table 1. List of all guinea pigs (Cavia porcellus) naturally infected with Leishmania enriettii, their gender, number of skin lesions, and town where they were raised in Paraná state, Brazil.

\begin{tabular}{llll}
\hline Animal identification & Sex & Number of lesions & \multicolumn{1}{c}{ Geographical location } \\
\hline CUR 1 & female & 04 & Campina Grande do Sul \\
CUR2 & female & 06 & Campina Grande do Sul \\
CUR 3 & female & 02 & Campina Grande do Sul \\
CUR 8 & female & 09 & Pinhais \\
CUR 9 & female & 03 & Quatro Barras \\
CUR10 & male & 01 & Campina Grande do Sul \\
CUR 61 & male & 05 & Curitiba, Santa Felicidade \\
CUR161 & female & 03 & Curitiba- Champagnat \\
CUR 173 & female & 04 & Curitiba- Champagnat \\
CUR 174 & male & 04 & Curitiba- Champagnat \\
CUR175 & male & 05 & Curitiba- Champagnat \\
CUR176 & female & 06 & Castro \\
CUR177 & male & 02 & Almirante Tamandaré \\
CUR178 & female & 02 & Mandirituba \\
CUR179 & female & 01 & Mandirituba \\
CUR180 & male & 01 & Mandirituba \\
CUR181 & male & 02 & Mandirituba \\
CUR185 & male & 02 & Mandirituba \\
CUR186 & female & 02 & Mandirituba \\
CUR188 & female & 02 & Curitiba, Mercês \\
CUR312 & female & 02 & Curitiba, Santa Felicidade \\
CUR325 & female & 05 & Laranjeiras do Sul \\
CUR326 & female & 08 & Laranjeiras do Sul \\
CUR357 & female & 04 & Curitiba, São Braz \\
CUR 360 & female & 04 & Curitiba, Santa Felicidade \\
CUR 361 & female & 01 & Curitiba, Santa Felicidade \\
\hline
\end{tabular}




\section{Parasite detection in guinea pigs and wild animals}

Amastigote forms of the parasite were observed by the direct exam of lesions from $100 \%$ of the 26 guinea pigs included in the study (Figure 2) by examining a stained slide containing the fine-needle aspirate, a lesion scraping or tissue imprint.

It was possible to in vitro grow and isolate promastigotes from 22 of the 26 animals. The in vitro culture from the other 4 animals also contained promastigotes but the culture got infected with yeast or bacteria and had to be eliminated before harvesting for DNA extraction.

The parasite was also isolated in culture from all internal organs examined. Equally, $100 \%$ of mucosae biopsies were positive.

None of the 77 wild animals captured showed amastigote forms by the direct exam or in vitro culture, except for Trypanosoma sp. that were isolated from six mouse-of-rice (Oryzomis sp), one porcupine (Sphiggurus villosus), and two marsupials.

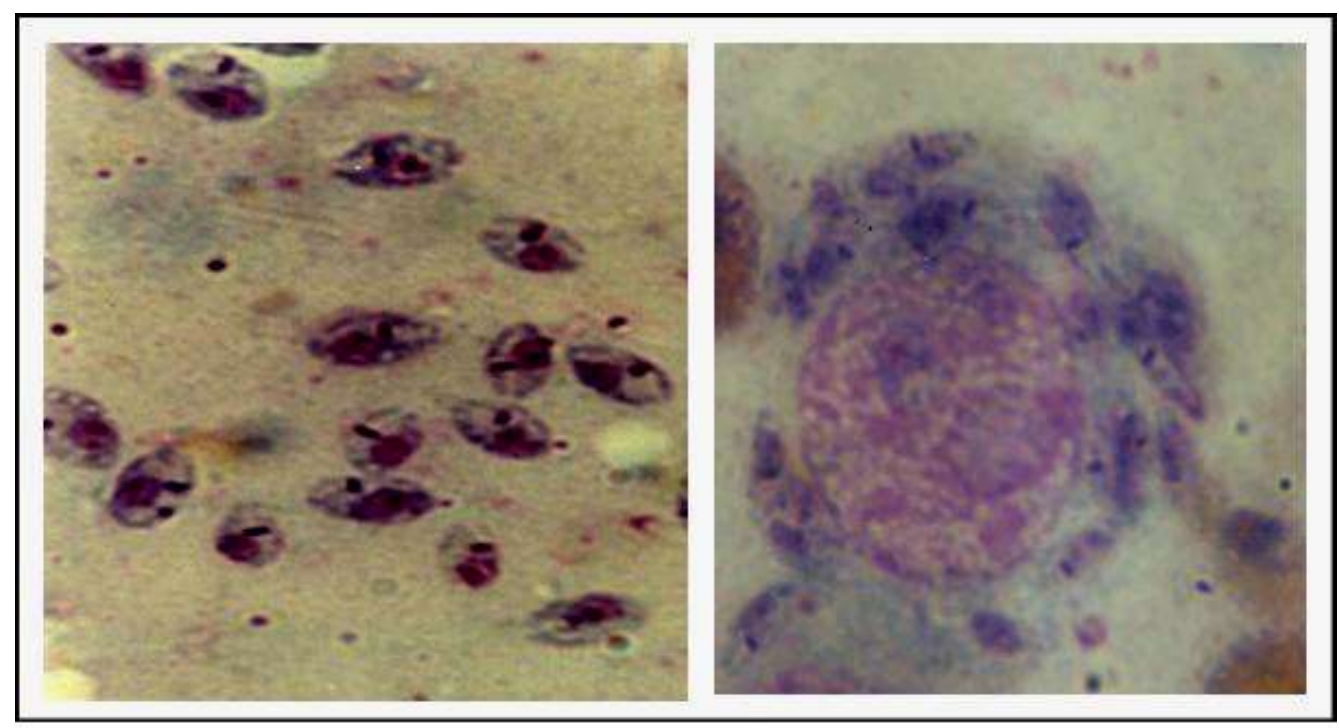

Figure 2. Leishmania enriettii amastigote forms obtained through the direct exam of a fine-needle aspirate from a guinea pig (Cavia porcellus) skin lesion, stained with May-Grünwald-Giemsa.

\section{Clinical description in guinea pigs}

We observed that the skin lesion starts as an area of alopecia and flaking of the skin, a condition that would later spread to other sites. The preference sites included the external aspect of the ears, nose, digits and less frequently the testicles and eyelids (Figure 3). Each lesion evolved rapidly into a reddish/brownish and hairless erythematous and edematous nodule. In approximately one month, the nodule would exudate a serosanguinous fluid and then ulcerate causing bleeding, resembling to some extent what have been described for human cutaneous leishmaniosis.

The active skin nodules were firm, with well-defined borders, and usually with blood crusts on and around the lesion. Some of the nodules would become larger than $5 \mathrm{~cm}$ in diameter and would show a warty aspect that when at the external ear caused it to bend down and over the ear canal. In 2-3 months, a few animals have developed disseminated lesions around the thoracic and abdominal area; however, these lesions appeared only as areas of alopecia with a crusty aspect, without formation of nodules.

The nodules around the digits could be large enough that would predispose the digits to trauma, fractures, nail avulsions, and also to the development of pododermatitis, especially when the animals were maintained in metal cages without padding. Other signs included hemorrhages from the lesions and lameness, worsening the clinical status.

Pruritus at the lesion site was hard to evaluate; nonetheless, head shaking suggested itching and discomfort, which augmented the bleeding from the nodule. We also checked for the presence of myiasis in and around the hemorrhagic foci but it was not detected.

The initial lesions occasionally healed; however, new wounds appeared in distinct locations. Growing of the hair or thickening of the skin was observed at a healed or healing lesion, as well as remaining crusts, but no erythema, ulcers, or hemorrhage. At this point, the animals were reaching the chronic stage of the disease 
where they were found sneezing and with epistaxis. Later they developed respiratory sounds, dyspnea, signs of airways obstruction, and would die from respiratory insufficiency. The survival time from the detection of the initial lesions to the animal's death could extend to over one year. This progression depended upon many factors, including age, nutritional status, and concomitant disorders.

Despite the undergoing pathologic events described above, the animals rarely showed fever, anemia, or acquired other infectious diseases. In case of a simultaneous debilitating disease the skin lesions were more aggressive and the clinical evolution was faster.

Regarding the behavior, all guinea pigs studied usually fed normally, independently of the severity of clinical signs, and did not show any behavioral changes until they reached the chronic stage of the disease. During this phase, they remained silent and lethargic certainly due to the respiratory impairment and pododermatitis.

\section{Genetic study of the parasite}

Through RAPD-PCR, several DNA fragments were amplified when using different primers. Considering that each primer has a different target sequence within the genome, the use of more than one primer was more efficient in detecting polymorphisms. While the use of primer A10 resulted in the amplification of amplicons of similar size throughout the samples (data not shown), other primers amplified fragments that were only seen in a few samples, characterizing the intra-species polymorphism. Regarding the A2 primer, five markers were amplified for most of the isolates, except CUR312 (Figure 4 A2 primer).

Using the A3 primer, 5 to 8 amplified fragments were seen in general for most isolates and for the $L$. enriettii reference strain. The amplified fragments for CUR61, CUR178 and CUR180 greatly differed from the other isolates; CUR178 and CUR180 showed a similar polymorphic pattern. The results for the $L$. braziliensis, $L$. amazonensis, and $L$. infantum reference strains differed significantly from all samples tested (Figure 4 A3 primer). The A9 primer generated 4 to 6 amplicons that repeatedly appeared across all isolates which were identified as $L$. enriettii when compared to the $L$. enriettii reference strain (Figure 4 A9 primer). The isolates CUR325 and CUR326 showed slightly distinct markers but all were much different than $L$. braziliensis, $L$. amazonensis, and $L$. infantum reference strains. Together, the results obtained by RAPDPCR using different primers indicated that all isolates contained $L$. enriettii DNA. In addition, there was intraspecific genetic variability as distinct amplified fragments were observed for each of the primers. 


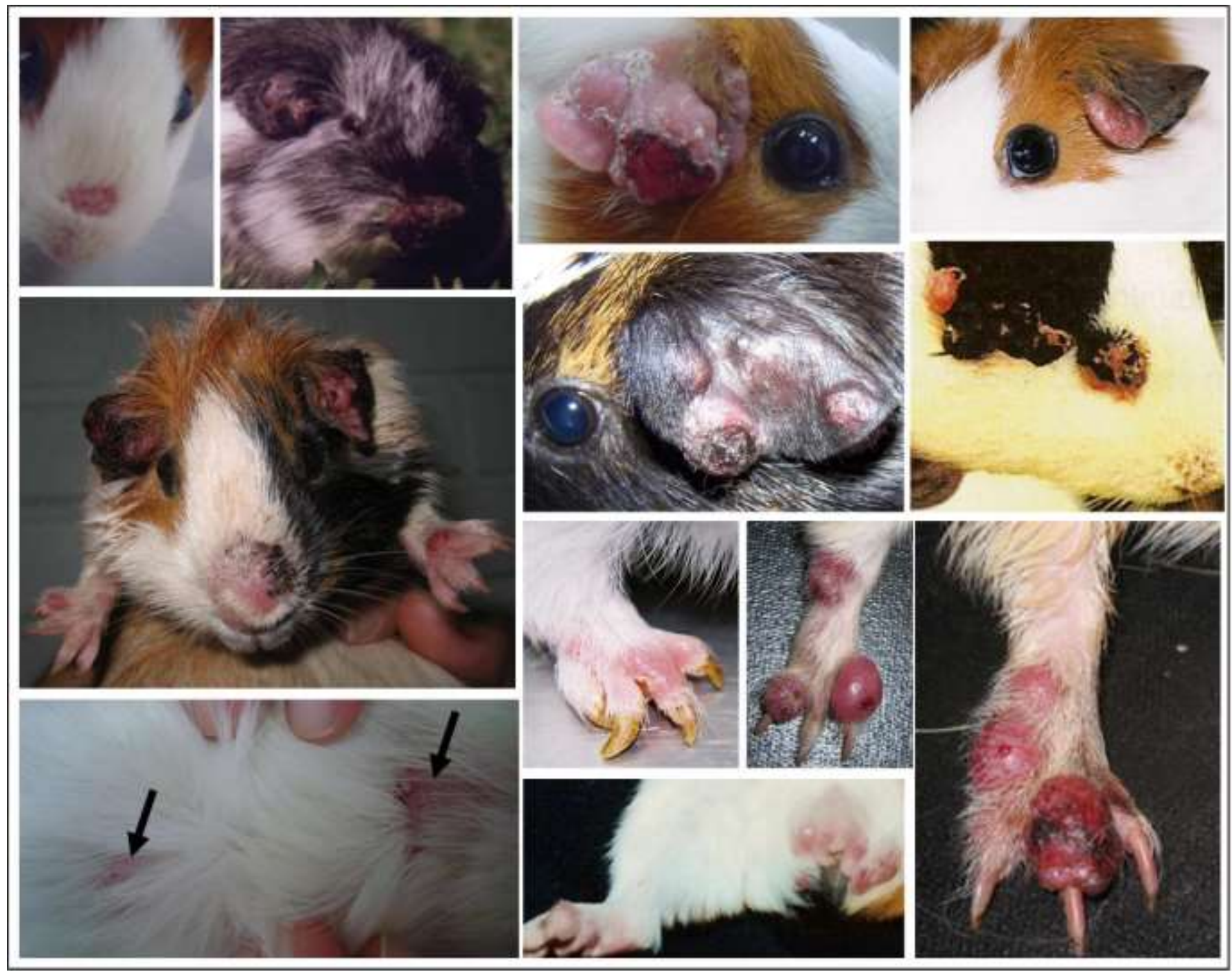

Figure 3. Skin lesions at distinct stages of evolution caused by Leishmania enriettii in guinea pigs (Cavia porcellus). Each lesion started as a hairless area with flaking of the skin and evolved to erythematous and edematous nodules. The preferred locations included the digits, ears, nose tip, eyelids, and testicles. Disseminated lesions with hairless and crusty aspect were found around the thoracic wall (arrows).

\section{Phenetic analysis}

The RAPD-PCR experiments allowed us to clearly differentiate $L$. enriettii from the other Leishmania species used as controls. All isolates were grouped with $L$. enriettii reference strain. CUR61 and CUR361 were the most dissimilar; however, both had amplified fragments specific to $L$. enriettii (Figure 5). 


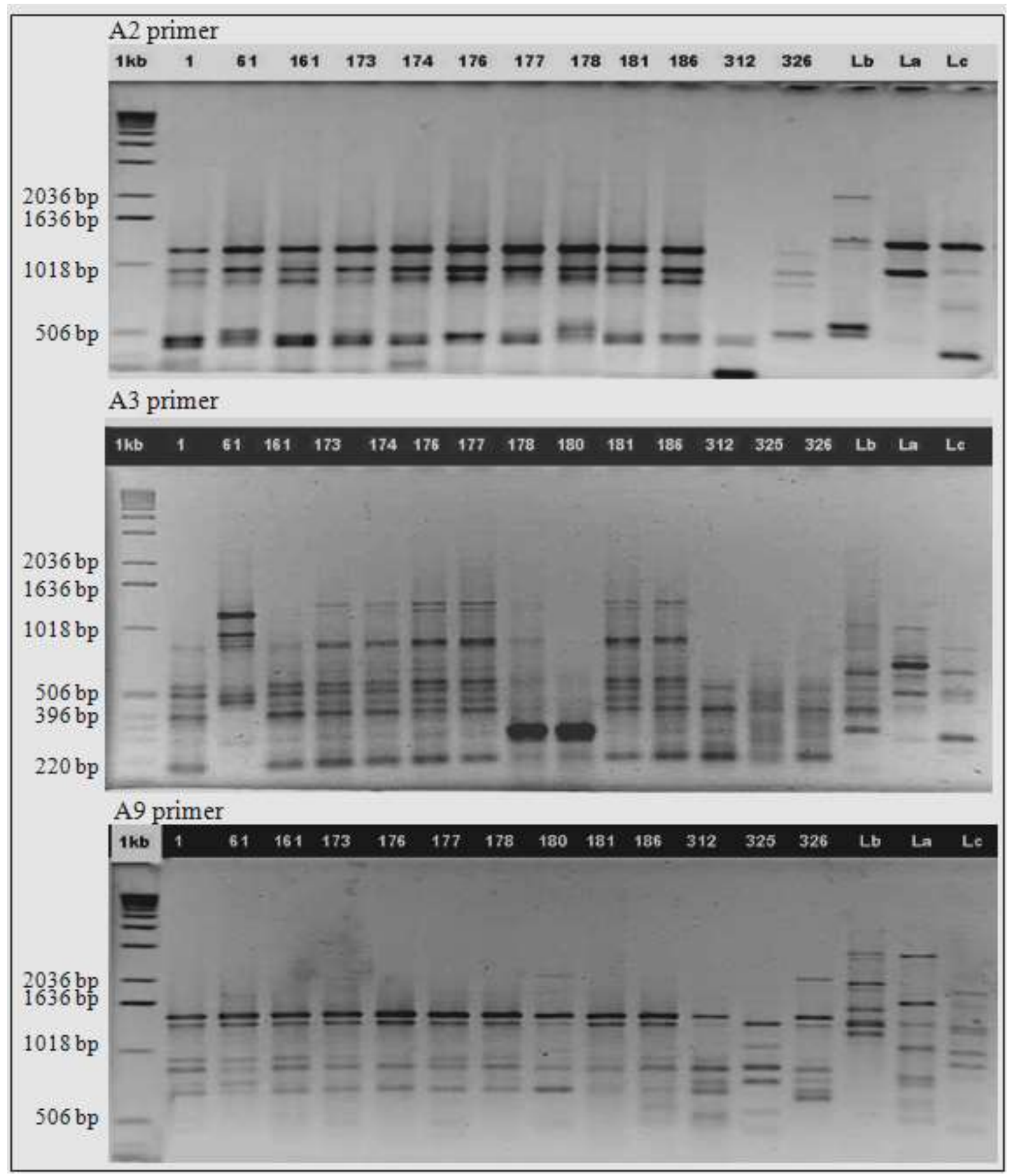

Figure 4. RAPD-PCR results characterizing Leishmania enriettii and the genetic variability among different isolates. Top, mid, and bottom panels are representative results obtained with the $\mathrm{A} 2, \mathrm{~A} 3$, and $\mathrm{A} 9$ primers, respectively. $1 \mathrm{~Kb}$ $1 \mathrm{~Kb}$ DNA ladder (Invitrogen) with sizes shown to the left. The numbers on the top of each panel indicate the different isolates shown on Table 1. 1- L. enriettii reference strain. $L b, L a$ and $L c$ are reference strains for $L$. braziliensis, $L$. amazonensis and $L$. chagasi syn. $L$. infantum. Three replicates were obtained for each primer. 


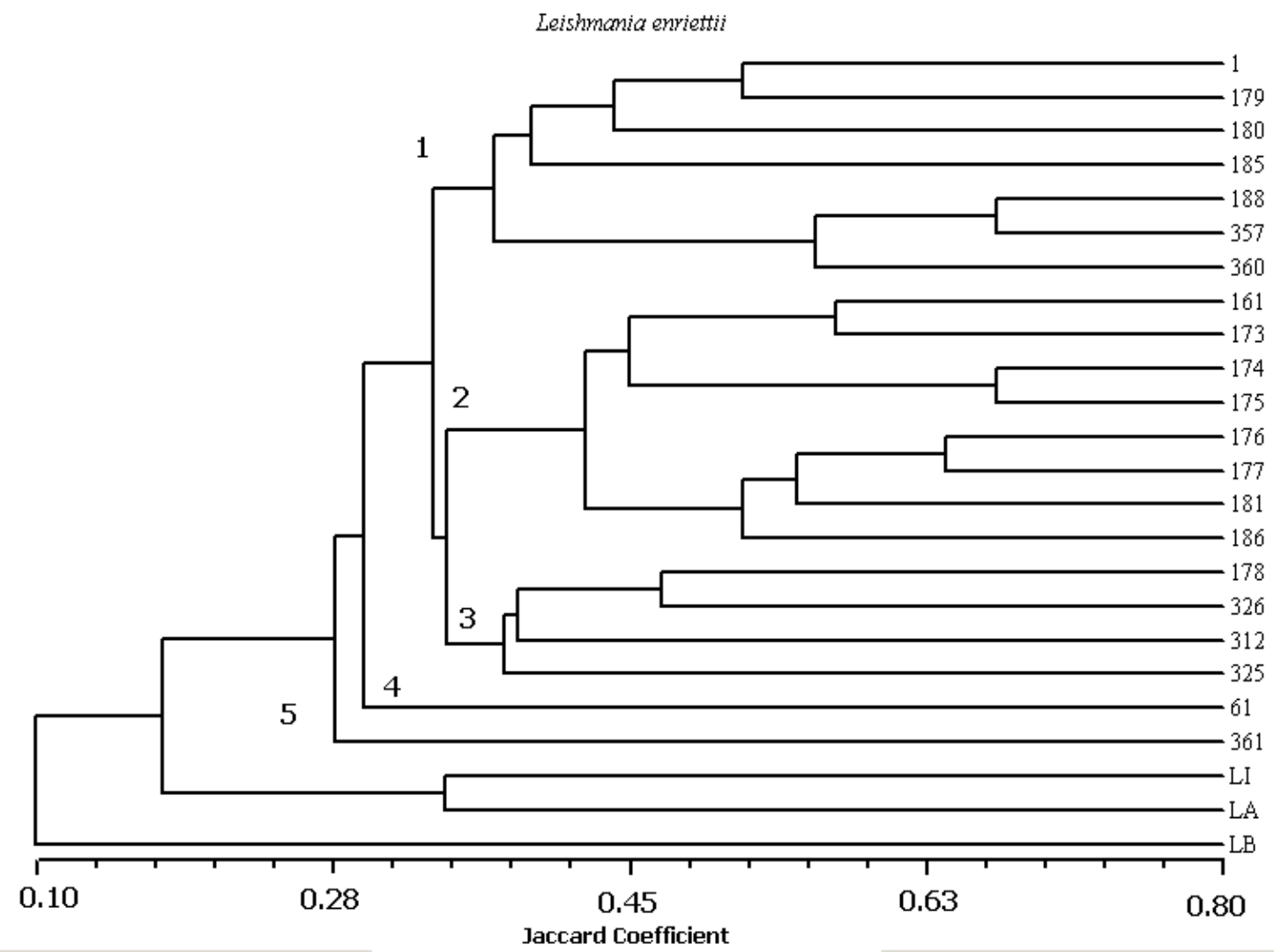

Figure 5. Dendrogram build for the guinea pig isolates of $L$. enriettii from Paraná state showing 5 clusters with $65 \%$ of similarity. The numbers to the right represent isolates from the animals listed on table 1 . Number 1 , on top indicates $L$. enriettii reference strain. LI, LA, LB are the reference strains for $L$. infantum, L. amazonensis and $L$. braziliensis, respectively. The RAPD-PCR raw data from the four primers (A2, A3, A9, and 10) was analyzed through the UPGMA method and the Jaccard coefficient.

\section{DISCUSSION}

Regarding the geographical distribution, most guinea pigs infected with $L$. enriettii from this study were from the state's capital Curitiba and its metropolitan area. Only two animals were from Laranjeiras do Sul and Castro cites. All guinea pig-owners asserted that the animals were born in Paraná, always lived there, and never had contact with forests and consequently with the insect vector, raising the hypothesis of transplacental transmission. Nonetheless, Paraná state offers favorable environmental conditions to the $L$. enriettii life cycle and perpetuation [17]. The outskirts of the capital Curitiba, where most infected guinea pigs were found at, is situated at the Iguaçu river basin. This region is characterized by a mean altitude of 600 to 800 meters, with a few places reaching $1000 \mathrm{~m}$. In addition, there are well distributed precipitations throughout the year. The relative air humidity correlates with the temperature and is influenced by the altitude. At this first plateau, also called Curitiba's plateau, the vegetation is mostly of araucaria moist forests and extends from the west of the Sea Mountain Ranges, near the ocean, through the second and third plateaus, near Laranjeiras do Sul. Similarly to the host enigma, the vectors for $L$. enriettii are still unknown. However, Paraná state (around Curitiba) harbors a sand fly that has been experimentally infected with $L$. enriettii and, thus, could sustain the life cycle of the parasite in the state $[15,17]$.

In addition to Curitiba and nearby cities, domestic guinea pigs naturally infected with $L$. enriettii were only found in two other Brazilian states, two animals in Capão Bonito, in the state of São Paulo [16], and 6 domestic guinea pigs from the state of Rio Grande do Sul $[26,27]$. Nothing was reported about the fauna and flora of those regions, suggesting that these isolated cases of leishmaniosis may represent allochthonous cases. Our observations indicate that southern Brazil, more specifically southwestern Paraná state, contains a favorable ecosystem for the existence and perpetuation of $L$. enriettii.

Most information on the clinical aspects of leishmaniosis in guinea pigs derives from experimental work $[2,6,28,29]$. Data from natural infections are more sporadic; however, the presence of nodular lesions observed mainly at the ears and nose of guinea pigs have been previously reported [1,16-17,26-27]. Although 
leishmaniosis caused by $L$. enriettii was described over 75 years ago, the nodular lesions are probably misdiagnosed as neoplasia or scabies. Thus, a better description of the chief clinical signs caused by the natural infection of $L$. enriettii in guinea pigs was needed to Veterinarians that should include leishmaniosis on their differential diagnosis of skin diseases.

After observing dozens of naturally infected guinea pigs from pet shops, or taken by veterinary hospitals, we have concluded that $L$. enriettii causes the diffuse cutaneous leishmaniosis (DCL), invading the mucous membranes and leading to a respiratory distress that causes the animal's death.

The DCL is a rare form of the disease also observed in anergic humans when infected by other species of Leishmania. The first description of human DCL was in 1946 in Venezuela [30]. Coincidently, this human DCL case was described at the same year $L$. enriettii was described in guinea pigs in Brazil. Convit and Lapenta [30] portrayed DCL as a peculiar form of leishmaniosis because of the nodular lesions full of amastigotes that disseminated around the body. The description in humans is similar to what we have observed in guinea pigs with the difference that in guinea pigs we observed invasion to the mucous membranes and death by respiratory insufficiency. In addition to Venezuela, human DCL has also been reported in other countries like in the United States (Texas), Mexico, Dominican Republic, Honduras, Colombia, Peru, Bolivia, and Brazil. In this case, the causative pathogens include L. (L.) pifanoi, L. (L.) mexicana, and $L$. (L.) amazonensis, which are phylogenetically close to L. enriettii [18].

In addition to lesions distributed throughout the body, L. enriettii DNA can be detected in different tissues [1,31] and this study corroborates the statement proposed by Medina [1] and Paraense [3] in which the parasite disseminates via the lymphatics and/or the bloodstream. Thus, L. enriettii and other species responsible for DCL are likely to disseminate similarly through the lymphatic and the hematogenous way.

The four primers were chosen to show the genetic variability in the genus Leishmania, as also described elsewhere [32]. Previously, Thomaz Soccol and coauthors, 1996 [17] isolated and characterized four strains of $L$. enriettii by isoenzymes, and found two zymodemes. With RAPD markers it was demonstrated a great variability within this species. As this parasite has been shown earlier in the lineage of the subgenus Leishmania [18] it will be important, in a second time, to sequence the isolates to confirm the genetic variability and the ancestry of this parasite. Interestingly, the phylogenetic analysis of the most recent Leishmania strains isolated in Australia revealed to be close to Leishmania enriettii [33].

In regards to the natural reservoirs for $L$. enriettii, guinea pigs have been pointed out as the unique candidates. However, because it develops the severe type of leishmaniosis, as described here, behaving like an anergic organism, it is unlikely to be a good reservoir based in the criteria proposed by WHO [34]. We have searched for natural hosts among several species of wild animals, by culturing of the parasite from internal organs or from skin biopsies, from non-lesion areas, and all tries were negative. Our results are supported by the findings of Muniz and Medina [2] that in the search for potential natural hosts, experimentally inoculated amastigotes or promastigotes in different animals (monkeys, laboratory mice, hamsters, rabbits, dogs, Cavia aperea, agoutis and even humans), however, all tries failed and none of the animals became infected, except for the guinea pigs.

Even though some groups were able to establish the experimental infection in hamsters $[35,36]$, others $[17,37]$ believe the enigmatic $L$. enriettii should have a sylvatic animal, mainly Cavia aperea, as the reservoir host. Machado and coauthors [16] captured apparently infected Cavia aperea near the Atlantic forest but could not isolate $L$. enriettii from any of the captured animals.

Ecco and coauthors [26] brings up a discussion suggesting $L$. enriettii as a cause of zoonosis; thus, if natural reservoirs do exist, it would be important to define and control them. However, from the data cited above we know that $L$. enriettii was never isolated from other animals and did not grow after being inoculated in humans. In addition, we have been working the past 30 years with leishmaniosis in animals and humans from Paraná state and the only autochthonous species isolated from humans until the present date is $L$. braziliensis. Therefore, it is unlikely that $L$. enriettii would be implicated with a zoonosis.

\section{CONCLUSION}

Leishmania enriettii shows genetic variability and is responsible for causing a diffuse cutaneous leishmaniosis in guinea pigs. It circulates in Curitiba and its metropolitan area which could be the center for dispersion of the species as it has already been found in other municipalities, suggesting the phenomenon of capture and installation of new foci. 
Acknowledgments: We wish to thank Dr. Medina who found and described Leishmania enriettii for the first time. He distributed the strain to various cryobanks around the world and this has allowed this species to be known all over the world. I had the thankful satisfaction of meeting him and telling him that he was known all over the world. His joy of knowing this prompted us to continue our research with this species. At the $50^{\text {th }}$ anniversary of BABT, he was still with us. Today, on the occasion of the $75^{\text {th }}$ anniversary of BABT, he is already a star in the sky.

Conflicts of Interest: The authors declare no conflict of interest.

\section{REFERENCES}

1. Medina H. Estudos sobre leishmaniose: I. Primeiros casos de leishmaniose espontânea observados em cobáios. Braz Arch Biol Technol. 1946;1:39-74.

2. Muniz J, Medina H. Leishmaniose tegumentar do cobaio (Leishmania enriettii n. sp.). Hospital (Rio de Janeiro) 1948;33:7-25.

3. Paraense WL. The spread of Leishmania enriettii through the body of the guinea pig. Trans R Soc Trop Med Hyg 1953;47:556-60.

4. Bryceson AD, Bray RS, Wolstencroft RA, Dumonde DC. Cell mediated immunity in cutaneous leishmaniasis of the guinea-pig. Trans R Soc Trop Med Hyg. 1970;64:472.

5. Kadivar DM, Soulsby EJ. Model for disseminated cutaneous leishmaniasis. Science. 1975;190:1198-200.

6. Kanan MW. Mucocutaneous leishmaniasis in guinea-pigs inoculated intravenously with Leishmania enriettii. Preliminary report. Br J Dermatol. 1975;92:663-73.

7. Mauel J, Behin R, Biroum N, Rowe DS. Mechanisms of protective immunity in experimental cutaneous leishmaniasis of the guinea-pig. I. Lack of effects of immune lymphocytes and of activated macrophages. Clin Exp Immunol. 1975;20:339-50.

8. Behin R, Mauel J, Rowe DS. Mechanisms of protective immunity in experimental cutaneous leishmaniasis of the guinea-pig. III. Inhibition of leishmanial lesion in the guinea-pig by delayed hypersensitivity reaction to unrelated antigens. Clin Exp Immunol. 1977;29:320-25.

9. Monroy A, Ridley DS, Heather CJ, Ridley MJ. Histological studies of the elimination of Leishmania enriettii from skin lesions in the guinea-pig. Br J Exp Pathol. 1980;61:601-10.

10. Mauel J, Behin R, Louis J. Leishmania enriettii: immune induction of macrophage activation in an experimental model of immunoprophylaxis in the mouse. Exp Parasitol. 1981;52:331-45.

11. Tobin JF, Laban A, Wirth DF. Homologous recombination in Leishmania enriettii. Proceedings of the National Academy of Sciences of the USA. 1991;88:864-68.

12. Wong IL, Chan KF, Zhao Y, Chan TH, Chow LM. Quinacrine and a novel apigenin dimer can synergistically increase the pentamidine susceptibility of the protozoan parasite Leishmania. J Antimicrob Chemother. 2009;63:1179-90.

13. Paranaiba LF, Pinheiro LJ, Torrecilhas AC, Macedo DH, Menezes-Neto A, Tafuri WL, et al. Leishmania enriettii (Muniz \& Medina, 1948): A highly diverse parasite is here to stay. PLoS Pathog. 2017;13(5):e1006303.

14. Paranaiba L, Pinheiro L, Macedo D, Menezes-Neto A, Torrecilhas A, Tafuri W, Soares R. An overview on Leishmania (Mundinia) enriettii: Biology, immunopathology, LRV and extracellular vesicles during the host-parasite interaction. Parasitology. 2018;145(10):1265-73.

15. Luz E, Giovannoni M, Borba A. Infecção de Lutzomyia monticola por Leishmania enriettii. Anais da Faculdade de Medicina da Universidade Federal do Paraná. Curitiba. 1967;121-16.

16. Machado MI, Milder RV, Pacheco RS, Silva M, Braga RR, Lainson R. Naturally acquired infections with Leishmania enriettii Muniz and Medina 1948 in guinea-pigs from Sao Paulo, Brazil. Parasitol. 1994;109:135-38.

17. Thomaz Soccol V, Pratlong F, Lange R, Castro E, Luz E, Dedet JP. New isolation of Leishmania enriettii Muniz and Medina, 1948 in Parana state, Brazil, 50 years after the first description, and isoenzymatic polymorphism of the $L$. enriettii taxon. Ann Trop Med Parasitol. 1996;90:491-95.

18. Thomaz Soccol V, Lanotte G, Rioux JA, Pratlong F, Martini-Dumas A, Serres E. Phylogenetic taxonomy of new world Leishmania. Ann Parasitol Hum Comp. 1993;68(2):104-6.

19. Castro EA, Thomaz Soccol V, Augur C, Luz E. Leishmania (Viannia) braziliensis: epidemiology of canine cutaneous leishmaniasis in the State of Parana (Brazil). Exp Parasitol. 2007;117:13-21.

20. Pereira EFA, Thomaz Soccol V, Lima HC, Thomaz Soccol A, de Castro EA, Mulinari-Brenner F, Queiroz-Telles F, Luz E. Molecular diagnosis of leishmaniosis in the Parana state of southern Brazil. Exp Dermatol. 2008;17:102430.

21. Sambrook J, Russel D. Molecular Cloning: a Laboratory Manual. New York: Cold Spring Harbor Laboratory Press; 2001. 
22. Thomaz Soccol V, Castro EA, Carvalho Y, Pereira EA, Szargiki R, Alcântara F, et al. A new focus of cutaneous leishmaniasis in the central area of the State of Paraná, south of Brazil. Acta Trop. 2009;111:308-15.

23. Miller M. TFPGA-Tools for population genetic analyses version 1.3. Flagstaff: Northern Arizona University; 1997.

24. Schluter $P$, Harris P. Analysis of multilocus fingerprinting data sets containing missing data. Mol Ecol Notes. 2006;6:569-72.

25. Swofford D. PAUP*: Phylogenetic analysis using Parsimony (and other methods) 4.0 Beta. Sunderland: Sinauer Associates; 2002.

26. Ecco R, Langohr I, Jew S, Barros S, Barros C. Cutaneous leishmaniasis in guinea pigs (Cavia porcellus). Cienc. Rural. 2000;30:525-28.

27. Rosa LD, Soares AG, Marcili A, Diaz JDS, Wolkmer P, Bassuino DM, et al. Cutaneous leishmaniasis in Cavia porcellus (guinea pig): case report. Arq Bras Med Vet Zootec. 2020;72(3).

28. Neal RA, Miles RA. Effect of sodium stibogluconate on infections of Leishmania enriettii, with observations on the interaction of drug and immune response. Ann Trop Med Parasitol. 1977;71:21-7.

29. El-On J, Witztum A, Schnur LF. Protection of guinea pigs against cutaneous leishmaniasis by combined infection and chemotherapy. Infect Immun. 1986;51:704-6.

30. Convit J, Lapenta P. Sobre um caso de leishmaniasis disseminada. Rev Pat Clin. 1946;17:153-8.

31. Sarnoski S. Aspectos patológicos na infecção natural e experimental de Leishmania enriettti (MUNIZ E MEDINA, 1948) em Cavia porcellus. Basic Pathology. Curitiba: Universidade Federal do Paraná; 1997.

32. Tibayrenc M, Neubauer K, Barnabé C, Guerrini F, Skarecky D, Ayala FJ. Genetic characterization of six parasitic protozoa: Parity between random-primer DNA typing and multilocus enzyme electrophoresis. Proc Natl Acad Sci U.S.A. 1993;90:1335-9.

33. Dougall A, Shilton C, Low Choy J, Alexander B, Walton S. New reports of Australian cutaneous leishmaniasis in Northern Australian macropods. Epidemiol Infect. 2009;137:1516-20.

34. WHO - World Health Organization. Control of the leishmaniases. Report of a meeting of the WHO Expert Committee on the Control of Leishmaniases, Geneva, 22-26 March; 2010.

35. Heisch RB, Killick-Kendrick R, Guy MW, Dorrell J. The development of trypanosomes, leishmaniae and ascitic tumour cells in the testicles of laboratory animals. Trans R Soc Trop Med Hyg. 1970;64:679-82.

36. Belehu A, Turk JL. Establishment of cutaneous Leishmania enriettii infection in hamsters. Infect Immun. 1976;13: 1235-41.

37. Lainson, R. On Leishmania enriettii and other enigmatic Leishmania species of the Neotropics. Mem Inst Oswaldo Cruz. 1997;92:377-87.

2021 by the authors. Submitted for possible open access publication under the terms and conditions of the Creative Commons Attribution (CC BY NC) license (https://creativecommons.org/licenses/by-nc/4.0/). 